\title{
Management of Patients with Urological Cancers in Turkey during the COVID-19 Pandemic: Recommendations of Uro-oncology Association
}

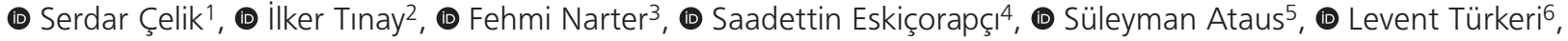 \\ (1) Sümer Baltacl ${ }^{7}$ \\ 1 University of Health Sciences Turkey, Izmir Bozyaka Training and Research Hospital, Clinic of Urology and Dokuz Eylül University Institute of Oncology, \\ Department of Basic Oncology, Izmir, Turkey \\ 2 Marmara University Faculty of Medicine, Department of Urology, Istanbul, Turkey \\ 3 Mehmet Ali Aydınlar University, Kadıköy Hospital, Clinic of Urology, Istanbul, Turkey \\ ${ }^{4}$ Mehmet Ali Aydınlar University, Atakent Hospital, Clinic of Urology, Istanbul, Turkey \\ 5 Forte Urology Center, Istanbul, Turkey \\ ${ }^{6}$ Mehmet Ali Aydınlar University, Altunizade Hospital, Clinic of Urology, Istanbul, Turkey \\ ${ }^{7}$ Ankara University Faculty of Medicine, Department of Urology, Ankara, Turkey
}

\begin{abstract}
Coronavirus disease-19 (COVID-19) has been declared as a pandemic by the World Health Organization. The number of cases has increased over time in correlation with the increasing number of testing worldwide. In order to use the available resources for COVID-19 treatment, urological practice has been limited only to emergency procedures and certain uro-oncological surgeries. In this report, we aim to share our recommendations for the daily uro-oncology practice in light of local circumstances in Turkey.
\end{abstract}

Keywords: COVID-19 pandemic, recommendations, urologic cancers, urologic oncology

\section{Introduction}

The human spread of a novel coronavirus, that is, Severe Acute Respiratory syndrome coronavirus 2, was first reported in Wuhan in December $2019(1,2)$. The disease was named coronavirus disease-19 (COVID-19), which is basically the short for "COVID-19" (2).

COVID-19 was declared as a pandemic by the World Health Organization on March 11, 2020, which is the same date on which the first confirmed case in Turkey was announced by the Ministry of Health (2). As in other countries, the number of cases has increased over time in correlation with the increasing number of testing in Turkey as well, and as of April 18, 2020, there were 82,329 confirmed cases in Turkey, ranking eighth after China worldwide.

Like in many countries, the urological practice in Turkey has also been limited only to emergency procedures and major uro-oncological surgeries in order to use the available resources for COVID-19 treatments. Although many sources have recently published the general recommendations for the daily practice in urological oncology, a potential concern is if these recommendations would be applicable to every patient from the different geographical regions of the world (3). While the duration of preventive measures, such as lockdowns or social isolations, remains unpredictable, it is of utmost importance that national organizations share their recommendations for daily practice based on national resources, sociocultural circumstances, and restrictive administrative regulations. In this report, we aim to share our recommendations for the daily urooncology practice in light of local circumstances in Turkey.

Urothelial Carcinoma (Bladder and Upper Urinary Tract)

a) Patient presented with hematuria and bladder mass

Cystoscopy and transurethral resection (TUR) can be performed as an outpatient procedure, and if the current conditions of the

Cite this article as: Çelik S, Tınay I, Narter F, Eskiçorapçı S, Ataus S, Türkeri L, Baltacı S. Management of Patients with Urological Cancers in Turkey during the COVID-19 Pandemic: Recommendations of Uro-oncology Association. Bull Urooncol 2020;19(3):100-103

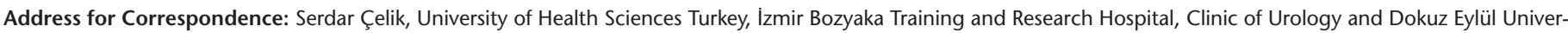
sity Institute of Oncology, Department of Basic Oncology, İzmir, Turkey Phone: +90 5057019631 E-mail: serdarcelik84@hotmail.com 
institution are appropriate, we do not recommend postponing this procedure. The current guidelines recommend a single intravesical instillation of chemotherapy in early postoperative period; however, owing to the potential complications of these intravesical instillations and the limited benefit, it is reasonable not to perform early postoperative instillations during COVID-19 pandemic $(4,5)$.

\section{b) Second TUR in patients with pT1 or high-grade urothelial carcinoma}

Residual disease can be found in $51 \%-58 \%$ of the patients, of which, $8 \%-11 \%$ are restaged to muscle-invasive disease after a second TUR $(6,7)$. To achieve better oncological outcomes, namely recurrence-free and progression-free survivals, the optimal duration of the second TUR is between two and six weeks after the initial resection (8). A second resection can be performed as an outpatient procedure, and if the current conditions of the institution are appropriate, we do not recommend postponing this procedure and perform it no longer than six weeks after the initial TUR. If there is a sufficient amount of muscle tissue in the specimen confirmed by pathological evaluation and the surgeon is confident of complete resection of all visible tumors, avoiding a re-TUR is an option during this period.

c) Intravesical BCG in patients with carcinoma in situ, pT1, or high-grade urothelial carcinoma

Intravesical Bacillus Calmette-Guérin (BCG) instillations can be performed as an outpatient procedure, and if the current conditions of the institution are appropriate, we do not recommend postponing this procedure since the risk of progression is high. Although, these instillations might cause serious complications, the potential benefits outweigh these risks.

However, intravesical treatment in low to moderate risk nonmuscle invasive bladder cancer (NMIBC) can be postponed during this pandemic.

\section{d) Follow-up of non-muscle invasive urothelial carcinoma of the bladder}

Patients with low and intermediate-risk tumors: Postponing follow-up cystoscopies in these patients for up to six months is reasonable during the COVID-19 pandemic.

Patients with high-risk tumors: Postponing follow-up cystoscopies in these patients might be reasonable during the COVID-19 pandemic. However, follow-up with urine cytology and imaging of the urinary tract might be offered as an alternative followup approach during this period. Nevertheless, we do not recommend postponing the first follow-up cystoscopy at the third month after the initial diagnosis and intravesical induction BCG treatment.

\section{e) Muscle invasive urothelial carcinoma of the bladder}

If the current conditions of the institution are appropriate, we recommend performing radical cystectomy in patients with muscle invasive disease. Delaying radical surgery for more than three months is associated with upstaging and lymph nodepositive disease, although there are conflicting reports on the overall survival with respect to the timing of radical cystectomy (within 90 days vs more than 90 days after the diagnosis) $(9,10,11,12,13)$. Also, cystectomy for BCG non-responsive highrisk NMIBC should not be delayed.

Generally, higher volumes of hospital and surgeon are associated with lower levels of peroperative and postoperative complications and better oncological results. Referring patients with muscle-invasive disease to high-volume centers might be reasonable during COVID-19 pandemic.

\section{f) Upper urinary tract urothelial carcinoma}

Diagnostic ureterorenoscopy and tumor sampling: Can be performed as an outpatient procedure, and if the current conditions of the institution are appropriate, we do not recommend postponing this procedure. However, with regard to the use of a single intravesical instillation of chemotherapy in early postoperative period, due to the potential complications of these intravesical instillations, it seems reasonable not to perform the early postoperative instillations during COVID-19 pandemic. Radical nephroureterectomy: If the current conditions of the institution are appropriate, we recommend performing radical surgery in patients with high-risk non-metastatic disease. Delaying radical surgery for more than 90 days is associated with worse disease-specific and progression-free survival (14). The current guidelines recommend a single intravesical instillation of chemotherapy in postoperative period; however, due to the potential complications of these intravesical instillations, it seems reasonable not to perform the early postoperative instillations during COVID-19 pandemic.

\section{Prostate Cancer}

\section{a) Screening}

It seems reasonable not to perform the routine screening with prostate specific antigen (PSA) and/or with digital rectal examination (DRE) during COVID-19 pandemic (15).

\section{b) Prostate biopsy}

If the current conditions of the institution are appropriate, we recommend performing prostate biopsy under local anesthesia in a selected group of patients who are admitted with high PSA levels and/or DRE findings compatible with locally advanced high-risk tumors. In all other clinical scenarios, it seems reasonable not to perform prostate biopsy during COVID-19 pandemic (15).

\section{c) Treatment of local and locally advanced prostate cancer}

Postponing follow-up visits, imaging modalities for staging, and treatment alternatives (surgery, radiotherapy, etc) in these patients for up to six months seems reasonable during COVID-19 pandemic $(16,17)$. Even in patients with asymptomatic unfavorable intermediate, high, and very high-risk disease, delaying the surgery for up to six months is not associated with deleterious results. However, we do recommend starting androgen deprivation therapy (ADT) during this waiting period. 


\section{d) Treatment of metastatic disease}

We recommend starting an ADT during COVID-19 pandemic in patients recently diagnosed with metastatic disease. It seems reasonable not to start with docetaxel chemotherapy during COVID-19 pandemic (18). However, if the current conditions of the institution are appropriate, we recommend offering orchiectomy with local anesthesia. The treatment of $150 \mathrm{mg}$ bicalutamide daily may be an alternative for hormonal therapy.

Patients who are undergoing an ADT might benefit from telemedicine, where they can share their PSA and testosterone results with the physician and decide together whether to pause or to continue with their treatment. Three or six monthly forms of luteinising hormone-releasing hormone agonist should be preferred in order to decrease patients' exposure to medical facilities. Moreover, as a second-line treatment alternatives, we recommend using enzalutamide, abiraterone, or apalutamide instead of docetaxel chemotherapy during COVID-19 pandemic.

\section{Kidney and Adrenal Tumors}

\section{a) Management of clinical stage $\mathrm{T} 1$ and $\mathrm{T} 2$ kidney tumors}

A three-month treatment delay in clinical stage $\mathrm{T} 1$ and $\mathrm{T} 2$ kidney tumors has no negative effect on survival (16). Therefore, it seems reasonable not to perform partial or radical nephrectomy during COVID-19 pandemic. However, in selective cases with CT1b and CT2 tumors and if the current conditions of the institution are appropriate, surgical approach might be offered as the treatment modality (17).

\section{b) Management of clinical stage T3 and T4 kidney tumors}

If the current conditions of the institution are appropriate, we recommend performing radical surgery in patients with clinical stage T3 and T4 kidney tumors. Postponing the treatment in these stages can decrease the survival rates and increase the surgery-related morbidities due to a possible risk of aggressive and progressive tumors in these stages $(16,17)$.

\section{c) Tumor biopsy in kidney tumors}

No recommendation on performing tumor biopsy prior to deciding on its postponement has been provided in the guidelines. However, it can be recommended to discuss with the patient on the diagnostic value of the tumor biopsy and the status of the center for this procedure and to determine the necessity of tumor biopsy in this regard. In metastatic patients, biopsy can be planned for tissue diagnosing before planning of systemic treatment.

\section{d) The case of kidney tumor-induced hemorrhage}

In case of tumor-related bleeding, an emergency radical nephrectomy can be performed according to the hemodynamic status of the patient, regardless of the tumor stage and metastasis status (18). However, primary efforts for embolization by an interventional radiologist seem reasonable during COVID-19 pandemic. e) Cytoreductive nephrectomy in patients with metastatic disease

The guidelines recommend delaying surgery in such patients with neoadjuvant therapy and minimizing the risk of patient's contact, especially those who will be undergoing a surgery (19). Moreover, in the current situation, in metastatic kidney tumors, a direct systemic treatment can be started instead of cytoreductive nephrectomy.

\section{f) Follow-up of patients on systemic therapy}

Oral (vascular endothelial growth factor-targeted therapy) treatment options might be preferred instead of intravenous therapy (checkpoint inhibitors). In addition, the use of prophylactic antibiotic and growth factor in patients under systemic therapy can reduce the potential morbidity by minimizing the risks of neutropenia and infection (19).

\section{g) Adrenal tumors}

If the current conditions of the institution are appropriate, we recommend performing adrenalectomy in patients who have $>6 \mathrm{~cm}$ adrenal mass or unfavorable radiological findings for adrenocortical carcinoma (17). In other adrenal tumors, it seems reasonable not to perform partial or radical adrenalectomy and to offer follow-up together with endocrinology clinic during COVID-19 pandemic.

\section{Testicular Cancer}

\section{a) Patient with a "suspected" testicular mass}

Radical orchiectomy can be performed as an outpatient procedure and patients can usually be discharged within 4 hours after the surgery with oral pain medication. We recommend performing orchiectomy if the present conditions of the institution are convenient to perform surgeries (20).

\section{b) Stage I seminoma}

We recommend using the risk-adapted approach. Patients without any risk factors (ie tumor size $>4 \mathrm{~cm}$ or stromal rete testis invasion) should be managed with surveillance. Additionally, while patients with only one risk factor can also be managed with surveillance according to the SWENOTECA trial results (21), patients with two risk factors should, however, be offered an adjuvant treatment. Considering the possible immunosuppressive effects of the chemotherapy agents, adjuvant radiotherapy to the retroperitoneal area using the irradiation dose of $20 \mathrm{~Gy}$ can be offered as a reliable alternative (22).

\section{c) Stage I non-seminoma}

We recommend using the risk-adapted approach. While patients without any risk factors (ie lymphovascular invasion) should be managed with surveillance, patients with lymphovascular invasion can be offered an adjuvant treatment with one course of bleomycin, etoposide and cisplatin (23). The potential pulmonary toxicity of bleomycin might be a limitation in the current circumstances. 


\section{d) Metastatic germ cell tumors}

The potential pulmonary toxicity of bleomycin might be a limitation in the current circumstances. For such patients with poor lung function, Cisplatin - Etoposide - Ifosfamide (PEI) protocol can be offered. In addition, the use of prophylactic growth factor should be offered in all patients with germ cell tumors receiving chemotherapy.

\section{e) Patient with post-chemotherapy residual retroperitoneal mass}

Considering the acceptable postoperative length of stay (3 to 6 days), we recommend performing retroperitoneal surgery if the present conditions of the institution are convenient to perform surgeries (24).

\section{Conclusion}

This report reflects the temporary recommendations for urooncological practice in Turkey during the first three months of the COVID-19 pandemic. It is of utmost importance that national organizations share their recommendations for daily practice based on national resources, sociocultural circumstances, and restrictive administrative regulations in order to interact with other societies during pandemic.

\section{Acknowledgements}

Publication: The results of the study were not published in full or in part in form of abstracts.

Contribution: There is not any contributors who may not be listed as authors.

Conflict of Interest: No conflict of interest was declared by the authors.

Financial Disclosure: The authors declared that this study received no financial support.

\section{Ethics}

Peer-review: Externally peer-reviewed.

\section{Authorship Contributions}

Concept: S.Ç., I.T., F.N., S.E., S.A., L.T., S.B., Design: S.Ç., I.T., F.N., S.E., Data Collection and Processing: S.Ç., I.T., F.N., S.E., Analysis and Interpretation: S.E., S.A., L.T., S.B., Literature Search: S.Ç., I.T., F.N., Writing: S.Ç., I.T., F.N., S.E.

\section{References}

1. Lu H, Stratton CW, Tang YW. Outbreak of pneumonia of unknown etiology in Wuhan, China: The mystery and the miracle. J Med Virol 2020;92:401-402.

2. WHO. Novel Coronavirus 2019. Available at: https://www. who.int/emergencies/diseases/novel-coronavirus-2019/ question-and-answers-hub/q-a-detail/q-a-coronaviruses

3. Burki TK. Cancer guidelines during the COVID-19 pandemic. Lancet Oncol 2020;21:629-630.

4. Oddens JR, van der Meijden AP, Sylvester R. One immediate postoperative instillation of chemotherapy in low risk Ta, $\mathrm{T} 1$ bladder cancer patients. Is it always safe? Eur Urol 2004;46:336-338.

5. Elmamoun $\mathrm{MH}$, Christmas TJ, Woodhouse CR. Destruction of the bladder by single dose Mitomycin $\mathrm{C}$ for low-stage transitional cell carcinoma
(TCC)--avoidance, recognition, management and consent. BJU Int 2014;113:34-38.

6. Cumberbatch MGK, Foerster B, Catto JWF, et al. Repeat Transurethral Resection in Non-muscle-invasive Bladder Cancer: A Systematic Review. Eur Urol 2018;73:925-933.

7. Naselli A, Hurle R, Paparella S, et al. Role of Restaging Transurethral Resection for T1 Non-muscle invasive Bladder Cancer: A Systematic Review and Metaanalysis. Eur Urol Focus 2018;4:558-567.

8. Baltacı S, Bozlu M, Yıldıım A, et al. Significance of the interval between first and second transurethral resection on recurrence and progression rates in patients with high-risk non-muscle-invasive bladder cancer treated with maintenance intravesical Bacillus Calmette-Guérin. BJU Int 2015;116:721-726.

9. Mmeje CO, Benson CR, Nogueras-González GM, et al. Determining the optimal time for radical cystectomy after neoadjuvant chemotherapy. BJU Int 2018;122:89-98.

10. Boeri L, Soligo M, Frank I, et al. Delaying Radical Cystectomy After Neoadjuvant Chemotherapy for Muscle invasive Bladder Cancer is Associated with Adverse Survival Outcomes. Eur Urol Oncol 2019;2:390-396.

11. Gore JL, Lai J, Setodji CM, et al. Mortality increases when radical cystectomy is delayed more than 12 weeks: results from a Surveillance, Epidemiology, and End Results-Medicare analysis. Cancer 2009;115:988-996.

12. Bruins HM, Aben KK, Arends T), et al. The effect of the time interval between diagnosis of muscle-invasive bladder cancer and radical cystectomy on staging and survival: A Netherlands Cancer Registry analysis. Urol Oncol 2016;34:166.e1-6.

13. Ayres $B E$, Gillatt $D, M c P h a i l ~ S$, et al. A delay in radical cystectomy of $>3$ months is not associated with a worse clinical outcome. BJU Int 2008;102:1045.

14. Waldert $\mathrm{M}$, Karakiewicz $\mathrm{Pl}$, Raman JD, et al. A delay in radical nephroureterectomy can lead to upstaging. BJU Int 2010;105:812-817.

15. Recommendations for Prostate Cancer Early Detection During the COVID-19 Pandemic. Available at: https://www.nccn.org/covid19/pdf/ NCCN_PCa_COVID_guidelines.pdf

16. Stensland KD, Morgan TM, Moinzadeh A, et al. Considerations in the triage of urological surgeries during the COVID-19 pandemic. Eur Urol 2020. doi:10.1016/j.eururo.2020.03.027. [Epub ahead of print]

17. Ficarra V, Novara G, Abrate A, et al. Urology practice during COVID-19 pandemic. Minerva Urol Nefrol 2020.

18. Alex Mottrie. EAU Robotic Urology Section (ERUS) guidelines during COVID-19 emergency. 2020. Available at: https://uroweb.org/ eau-robotic-urology-section-erus-guidelines-during-covid-19-emergency/

19. Gillessen S, Powles T. Advice Regarding Systemic Therapy in Patients with Urological Cancers During the COVID-19 Pandemic. Eur Urol. 2020. doi: 10.1016/j.eururo.2020.03.026. [Epub ahead of print]

20. Huyghe E, Muller A, Mieusset R, et al. Impact of diagnostic delay in testis cancer: results of a large population-based study. Eur Urol 2007; 52:1710-1716.

21. Tandstad T, Ståhl $\mathrm{O}$, Dahl $\mathrm{O}$, et al. Treatment of stage I seminoma, with one course of adjuvant carboplatin or surveillance, risk-adapted recommendations implementing patient autonomy: a report from the Swedish and Norwegian Testicular Cancer Group (SWENOTECA). Ann Oncol 2016;27:1299-1304.

22. Jones WG, Fossa SD, Mead GM, et al. Randomized trial of 30 versus 20 $G y$ in the adjuvant treatment of stage I Testicular Seminoma: a report on Medical Research Council Trial TE18, European Organisation for the Research and Treatment of Cancer Trial 30942 (ISRCTN18525328). J Clin Oncol 2005;23:1200-1208.

23. Tandstad T, Ståhl O, Håkansson U, et al. One course of adjuvant BEP in clinical stage I nonseminoma mature and expanded results from the SWENOTECA group. Ann Oncol 2014;25:2167-2172.

24. Bourgade V, Drouin SJ, Yates DR, et al. Impact of the length of time between diagnosis and surgical removal of urologic neoplasms on survival. World I Urol 2014;32:475-479. 